\title{
Comparison of the performance of three cancer antigen (CA) 15-3 immunoassays
}

Nafija Serdarević ${ }^{1,2 *}$, Raif Serdarevićs ${ }^{3}$ Amra Memić ${ }^{4}$

${ }^{1}$ Institute for Clinical Chemistry and Biochemistry, Clinical Center University of Sarajevo, Sarajevo, Bosnia and Herzegovina, ${ }^{2}$ Faculty of Health Sciences, Sarajevo, Bosnia and Herzegovina, ${ }^{3}$ Department of Ophthalmology, Clinical Center University of Sarajevo, Sarajevo, Bosnia and Herzegovina, ${ }^{4}$ Department of Psychiatry, Clinical Center University of Sarajevo, Sarajevo, Bosnia and Herzegovina

\begin{abstract}
Introduction: In the present study we investigated the performance, precision, and recovery of three different automated methods in determining cancer antigen (CA) 15-3 levels.

Methods: Serum samples were obtained from 60 hospitalized female patients. As controls, commercially available samples were used. Cancer antigen (CA) 15-3 levels were measured using ARCHITECT CA 15-3, Elecsys ${ }^{\circ}$ CA 15-3, and Vitros CA 15-3 immunoassays. A comparison of the results between the three methods was conducted, and the precision and recovery were analyzed.
\end{abstract}

Results: Coefficient of variations (CVs), determined with low- and high-level-CA 15-3 control samples, and reproducibility values were: $2.56-2.80 \%$ and $3.10-4$. 20\% for ARCHITECT i2000SR immunoassay analyzer; 3.50-5.55\% and 4.88-6.47\% for Cobas E 601 analyzer; 3.30-4.0\% and $4.30-4.80 \%$ for VITROS 5600 Integrated System, respectively. The percent recoveries were 95-98\% for Elecsys ${ }^{\circ}$ CA $15-3$ assay, 93-105\% for Vitros CA 15-3 assay, and 92-95\% for ARCHITECT CA 15-3 assay. Method comparison results demonstrated correlation coefficient $(r)$ in range from 0.994 to 1 . The average CA 15-3 concentrations measured by Vitros, ARCHITECT, and Elecsys ${ }^{\circ}$ were $157.24+/-329.75 \mathrm{U} / \mathrm{mL}, 100.91+/-213.75 \mathrm{U} / \mathrm{mL}$, and $80.93+/-173.29 \mathrm{U} / \mathrm{mL}$, respectively.

Conclusions: Tumor marker CA 15-3 in individual patients should be monitored using the same immunoassay method, reagents, and analyzer. Different immunoassays tested on different analyzers, often show large discrepancies in reported values for individual patients. Different immunoassay technologies quantify analytes of clinical interest using monoclonal or polyclonal antibodies. Thus, the usage of antibodies with different specificities could explain the differences in CA 15-3 serum values between different methods.

Keywords: CA 15-3; immunoassays; chemiluminescent microparticle immunoassay; electro-chemiluminescence; Intellicheck technology

*Corresponding author: Nafija Serdarević,

Institute for Clinical Chemistry and Biochemistry, Clinical Center University of Sarajevo, Bolnička 25, Sarajevo, Bosnia and Herzegovina. E-mail: serdarevicnafija@yahoo.com

Submitted: 22 October 2016/Accepted: 01 December 2016

DOI: http://dx.doi.org/10.17532/jhsci.2016.381

UNIVERSITY OF SARAJEVO FACULTY OF HEALTH STUDIES

\section{INTRODUCTION}

Cancer antigen (CA) 15-3 is a glycoprotein with a high molecular weight $(>400 \mathrm{kDa})$, which belongs to a subgroup of polymorphic epithelial mucins (PEM). CA 15-3 is recognized by two monoclonal antibodies: DF3 (developed against 
membrane-enriched human breast cancer with metastases in the liver), and 115D8 (developed against milk fat globule membrane of human origin). Circulating DF3-reactive antigen has a molecular weight of $300-450 \mathrm{kDa}(1,2)$. Increased concentrations of CA 15-3 have been observed in various malignancies; nevertheless, it is the most useful marker in monitoring the disease progression and therapy success in patients with metastatic breast cancer. Measurement of CA 15-3 levels in the serum can also be used for the surveillance of patients diagnosed with breast cancer. Serial determinations are commonly used to monitor the treatment response in patients with advanced disease and also to increase the chance to detect early recurrence of the cancer. Increased serum concentrations of CA 15-3 (higher than $25 \mathrm{kU} / \mathrm{L}$ ) are observed in about $69 \%$ of patients with metastatic breast cancer, while only in $13 \%$ of patients with primary breast cancer. In rare cases, increased concentrations of CA 15-3 can be observed in patients with benign disease, e.g., benign liver diseases and benign diseases of the breast. As a tumor marker, CA 15-3 is not just specific to breast cancer. An increase in CA 15-3 serum level can also be observed in other tumors such as ovarian cancer, endometrial tumors, and lung cancer $(3,4)$. In general, procedures for measuring tumor markers differ between different manufactures. Because majority of antibodies used in various assays are obtained from common sources, there is a common misconception that the measurement results are equivalent between different methods $(5,6)$. However, it is well-known that repetitive sampling using different methods may show large variations in results. If this is not taken into account, inappropriate clinical decisions can be made. Thus, any changes in analytical methodology over last 6 months should be reported along with the measurement results and reference ranges $(5,7,8)$.

\section{METHODS}

\section{Patients}

This prospective study was conducted from January 2016 to May 2016 and included 60 female patients aged between 52 and 90 years, hospitalized at the Oncology Clinic, University Clinical Center
Sarajevo. The study protocol followed the ethical guidelines provided in the Declaration of Helsinki, and informed consent was obtained from all participants.

Blood samples were collected in $3.5-\mathrm{mL}$ serum separator tubes (Vacutainer ${ }^{\ominus}$, Becton Dickinson, Rutherford, NJ 07,070 U.S.). We used test tubes with clot activator and gel separator. Serum samples were obtained by centrifugation at $3000 \mathrm{rpm}$ using a SIGMA 3-16P device (SIGMA Laborzentrifugen GmbH; Osterode am Harz; Germany). After that, the serum concentration of CA $15-3$ was determined.

\section{Patient inclusion and exclusion criteria}

Clinical status of patients was evaluated by oncological examination, medical imaging, pathological analysis, and laboratory tests. Cancer-related events during the follow-up were documented using at least one imaging technique (i.e., X-ray or CT scan) or by puncture cytology, biopsies, and pathological examination following surgical removal. As part of clinical investigation, the serum concentrations of CA 15-3 were determined after mastectomy, in patients with breast cancer, at the Institute for Clinical Chemistry and Biochemistry, University Clinical Center Sarajevo. Because vitamin B12 deficiency and renal failure are common causes of increased CA 15-3 serum levels, patients with these conditions were excluded from the study. For the same reason, patients with pancreatitis, Crohn's disease, ulcerative colitis, benign gastrointestinal tumors, as well as menstruating women, were excluded from the study. Furthermore, patients with hemolysis, icterus, and lipemia were also excluded from the study. Additional exclusion criteria were: Pregnancy, related benign breast, inflammatory, infectious or autoimmune diseases, and significant metabolic alterations.

\section{Serum samples}

The serum samples were collected, aliquoted, and stored up to 8 days at $20^{\circ} \mathrm{C}$. To avoid the effects of thawing, a set of 50 aliquots per assay was available for each analyzer to perform correlation analyses. All samples were stored in the same manner, to avoid variation in storage conditions. The samples 
were thawed and homogenized immediately before conducting the experiment. According to the manufacturer instructions, the serum samples were free of fibrin and red blood cells. If testing was delayed more than 24 hours, the serum samples were removed from the clot, serum separator or red blood cells, and stored up to 7 days at $2-8^{\circ} \mathrm{C}$ prior to being tested. If testing was delayed more than 7 days, the serum samples were stored frozen at $-20^{\circ} \mathrm{C}$ or lower temperature (9).

\section{Chemiluminescent microparticle immunoassay (CMIA)}

CMIA is a noncompetitive sandwich assay for measuring analytes of interest. The amount of generated signal is directly proportional to the amount of analyte present in the sample.

Architect CA 15-3 asssay is a two-step immunoassay, based on CMIA technology, used to determine the presence of CA 15-3 in human serum. First, the sample, assay diluent and anti- CA 15-3-antibody-coated paramagnetic particles are combined. CA 15-3, present in the sample, binds to the anti-CA 15-3 coated microparticles. After incubation and wash, anti-CA 15-3-acridinium-labeled conjugat is added in the second step. Following another incubation and wash, pre-trigger and trigger solutions are then added to the reaction mixture. The pre-trigger solution (hydrogen peroxide) creates an acidic environment to prevent early release of energy (light emission), helps in keeping microparticles from clumping and splits acridinium dye off the conjugate bound to the microparticle complex (this action prepares the acridinium dye for the next step). The trigger solution (sodium hydroxide) dispenses to the reaction mixture. The acridinium undergoes an oxidative reaction when is exposed to peroxide and an alkaline solution. This reaction causes the occurence of chemiluminescent reaction. $\mathrm{N}$-methylacridone forms and releases energy (light emission) as it returns to its ground state. The resulting chemiluminescent reaction is measured as relative light units (RLU). A direct relationship exists between the amount of CA 15-3 in the sample and RLU detected by Architect System optics. The cut-off value range for measuring CA 15-3 by ARCHITECT i2000SR immunoassay analyzer (Abbot, Abbot Park, Illinois, USA) is $0-31.3 \mathrm{U} / \mathrm{mL}(9,10)$.

\section{Electro-chemiluminescence (ECLIA)}

ECLIA is Roche's technology for immunoassay detection of CA 15-3 in human serum. The development of ECLIA immunoassays is based on the use of a ruthenium-complex and tripropylamine (TPA). The chemiluminescence reaction for the detection of the reaction complex is initiated by applying a voltage to the sample solution resulting in a precisely controlled reaction. ECLIA technology can accommodate many immunoassay principles while providing superior performance. The cut-off value range for measuring CA 15-3 with Cobas E 601 analyzer (Roche Diagnostics, Basel, Switzerland), is 0-25 U/mL (11).

\section{Integrated System - Intellicheck ${ }^{\circledR}$ Technology}

The VITROS CA 15-3 test is performed using the VITROS CA 15-3 Reagent Pack and the VITROS CA 15-3 Calibrators. A two-step immunometric technique is used, which involves the reaction of CA 15-3 present in the sample with a biotinylated antibody (sheep polyclonal anti- CA 15-3) in the first step. The antigen-antibody complex is captured by streptavidin coated on the well. Unbound materials are removed by washing. The second step involves the reaction of antigen-antibody complex with a horse radish peroxidase (HRP)-labeled antibody conjugate (mouse monoclonal anti- CA 15-3). Unbound materials are removed by washing. The bound HRP conjugate is measured by a luminescent reaction. A reagent containing luminogenic substrates (a luminol derivative and a peracid salt) and an electron transfer agent, is added to the wells. The HRP in the bound conjugate catalyzes the oxidation of the luminol derivative, producing light. The electron transfer agent (a substituted acetanilide) increases the level of light produced and prolongs its emission. The light signals are read by the system. The amount of HRP conjugate bound is directly proportional to the serum concentration of CA 15-3. The cut-off value range for measuring CA 15-3 by VITROS 5600 Integrated System (Ortho Clinical Diagnostics, Raritan, NJ, USA) is 0-35 U/mL (12).

\section{Imprecision}

Commercial control samples with low and high CA 15-3 levels, for ARCHITECT i2000SR 
immunoassay analyzer, Cobas E 601 analyzer, and VITROS 5600 Integrated System, were used. The precision (intra-assay variation) was determined by measuring low and high values of the CA 15-3 controls $(n=20)$. The reproducibility (inter-assay variation) was tested with the same control sample for each test, once a day, over 10 consecutive days. Recovery studies were performed with ARCHITECT CA 15-3, Elecsys ${ }^{\oplus}$ CA 15-3, and Vitros CA 15-3 assays. Method comparison was performed using the serum samples of 60 patients referred to the laboratory for CA 15-3 measurement. Analyses of serum CA 15-3 levels were performed on ARCHITECT i2000SR, Cobas E 601, and VITROS 5600 Integrated System.

\section{Recovery analysis}

The recovery analysis was performed according to the principles given in Tietz Textbook of Clinical Chemistry (13). The serum concentrations of CA 15-3 were determined on the Cobas E 601, VITROS 5600 Integrated System, and ARCHITECT i2000SR immunoassay analyzer with the CA 15-3 immunoassays, and the percent of recovery was calculated. The DF3 defined antigen CA 15-3 in concentration of $45 \mathrm{U} / \mathrm{mL}$ (Epitope DTRPAP- Fujirebio Diagnostics, Inc Malvern, PA USA) was added to human serum samples.

\section{Statistical analysis}

The results were analyzed using SPSS version 16.0 software (SPSS Inc, Chicago, USA), and reported as average values $(\overline{\mathrm{x}})$, standard deviations (SDs),
Pearson correlation coefficient ( $r$ ), and equations of linear regression. The difference between the samples was analyzed using the $t$-test, with a statistical significance level set at $p<0.05$.

\section{RESULTS}

\section{Quality control testing}

The two types of controls $(n=20)$, with low and high levels of CA 15-3, were used for quality control testing. The results of quality control testing for the three immunoassays are shown in Table 1.

\section{Recovery}

The measured amounts of CA 15-3 were added to normal human serum samples. The mean recovery of CA $15-3$ assay was $100 \pm 15 \%$. The results are shown in Table 2.

\section{Method comparison results}

A comparison of CA 15-3 results in 60 serum samples obtained on the ARCHITECT i2000SR and Cobas E 601 analyzers was performed. The method comparison analysis demonstrated varying degrees of agreement between the assays performed on the ARCHITECT i2000SR and Cobas E 601 analyzers with slopes ranging from 0.77 to 0.83 , y-intercepts ranging from -6.66 to $5.78 \mathrm{U} / \mathrm{mL}$, and correlation coefficients ranging from 0.9899 to 0.9971 . The $t$-test results revealed a statistically significant difference between the two methods $(p=0.0059)$, and poorer agreement with the ARCHITECT i2000SR.

TABLE 1. Quality control testing

\begin{tabular}{|c|c|c|c|c|}
\hline Spiked concentration $(\mathrm{U} / \mathrm{mL})$ & $\begin{array}{c}\text { Intra-assay } \\
\text { concentration (mean } \\
\text { SD, } \mathrm{n}=20)(\mathrm{U} / \mathrm{mL})\end{array}$ & $\begin{array}{l}\text { Intra-assay } \\
\text { precision } \\
(\%) \\
\end{array}$ & $\begin{array}{c}\text { Inter-assay } \\
\text { concentration (mean } \\
\text { SD, } n=20)(U / m L)\end{array}$ & Reproducibility (\%) \\
\hline \multicolumn{5}{|c|}{ Elecsys $^{\circledast}$ CA 15-3 assay (ECLIA technology) } \\
\hline 16.0-24.4 & $19.47 \pm 0.65$ & 3.50 & $19.02 \pm 0.93$ & 4.88 \\
\hline $81.4-125$ & $99.01 \pm 5.50$ & 5.55 & $94 \pm 6.08$ & 6.47 \\
\hline \multicolumn{5}{|c|}{ Vitros CA 15-3 assay (Intellicheck technology) } \\
\hline $10.2-19.8$ & $15.1 \pm 0.50$ & 3.30 & $16.0 \pm 0.70$ & 4.30 \\
\hline $96-178$ & $138 \pm 4.60$ & 4.0 & $150 \pm 7.20$ & 4.80 \\
\hline \multicolumn{5}{|c|}{ ARCHITECT CA 15-3 assay (CMIA technology) } \\
\hline $12.5-18.70$ & $15.60 \pm 0.40$ & 2.56 & $16.10 \pm 0.5$ & 3.10 \\
\hline $73.60-110.0$ & $92 \pm 1.6$ & 2.8 & $95 \pm 4.00$ & 4.20 \\
\hline
\end{tabular}

ECLIA: Electro-chemiluminescence; CMIA: Chemiluminescent microparticle immunoassay; CA: Cancer antigen 
TABLE 2. Recovery testing

\begin{tabular}{|c|c|c|c|c|}
\hline Sample type & Endogenouslevel (U/mL) & Added CA 15-3 (U/mL) & Observed CA 15-3 (U/mL) & Percent recovery \\
\hline \multicolumn{5}{|c|}{ Elecsys $^{\circledR}$ CA $15-3$ assay (ECLIA technology) } \\
\hline 1 & 29.5 & 45 & 74 & 98 \\
\hline 2 & 30 & 45 & 72 & 96 \\
\hline 3 & 31.3 & 45 & 73 & 95 \\
\hline \multicolumn{5}{|c|}{ Vitros CA 15-3 assay (Intellicheck technology) } \\
\hline 1 & 35 & 45 & 85 & 105 \\
\hline 2 & 34.6 & 45 & 83 & 104 \\
\hline 3 & 40.2 & 45 & 80 & 93 \\
\hline \multicolumn{5}{|c|}{ ARCHITECT CA 15-3 assay (CMIA technology) } \\
\hline 1 & 30.00 & 45 & 72 & 96 \\
\hline 2 & 32.3 & 45 & 71 & 92 \\
\hline 3 & 33.2 & 45 & 75 & 95 \\
\hline
\end{tabular}

ECLIA: Electro-chemiluminescence; CMIA: Chemiluminescent microparticle immunoassay; CA: Cancer antigen

The results of method comparison analysis are shown in Figure 1.

The ARCHITECT i2000SR and VITROS 5600 Integrated System, with a slope ranging from 1.54 to 1.54 (95\% confidence interval [CI]) and correlation coefficients $\mathrm{r}=1.0$, demonstrated the highest degree of agreement. The analysis showed an intercept ranging from 1.30 to $1.79 \mathrm{U} / \mathrm{mL}$ (95\% CI). According to the $t$-test results, the difference between the methods was statistically significant $(p=0.0027)$. The comparison between the ARCHITECT i2000SR and VITROS 5600 methods is shown in Figure 2.

The method comparison of the assays performed on the Cobas E 601 and VITROS 5600 Integrated System showed a slope ranging from 0.50 to 0.53 (95\% CI), correlation coefficients ranging from 0.9900 to 0.9971 , and $y$ - intercept ranging from -7.46 to $4.94 \mathrm{U} / \mathrm{mL}$ (95\% CI). The difference between the methods was statistically significant ( $p=0.0029)$, according to the $t$-test results. The comparison between the Cobas E 601 and VITROS 5600 methods is shown in Figure 3.

The results for all assays were classified as normal or increased, and the analytical concordance was assessed using the cut-off values recommended by the manufacturer of each assay. The concentration range of CA 15-3 in the serum samples measured using the Cobas E 601 technology was 5.9-817.4 U/mL, while the CA 15-3 concentration range obtained with the ARCHITECT i2000SR

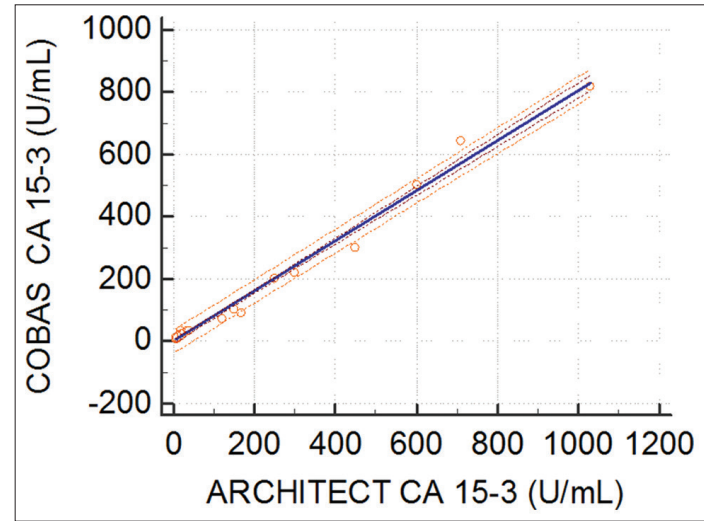

FIGURE 1. Comparison of cancer antigen (CA) 15-3 antigen determined in human serum samples using ARCHITECT i2000SR immunoassay analyzer and Cobas E 601 analyzer $(r=0.9899-0.9971)$.

technology was higher $(8.2-1030.2 \mathrm{U} / \mathrm{mL})$. The concentration range of $\mathrm{CA} 15-3$ in the serum samples determined using the VITROS 5600 Integrated System was 10.79-1010.96 U/mL. In our study, the serum concentrations of CA 15-3 measured by the VITROS 5600 Integrated System were significantly higher compared to the other two technologies, Cobas E 601 and ARCHITECT i2000SR. The average CA 15-3 concentrations measured by the VITROS, ARCHITECT and Cobas methods were $157.24+/-329.75 \mathrm{U} / \mathrm{mL}, 100.91$ $+/-213.75 \mathrm{U} / \mathrm{mL}$, and $80.93+/-173.29 \mathrm{U} / \mathrm{mL}$, respectively. The evaluation of method comparison analysis using Bland-Altman plot, to test the 
limits of agreement $(-1.96 s$ to $+1.96 s)$ between the three methods, is shown in Figures 4 and 5. The bias (mean difference) between the ARCHITECT i2000SR and Cobas E 601 was - $20 \mathrm{U} / \mathrm{mL}$, almost constant for all the measured concentrations, with the exception of very low values. The bias (mean difference) between the ARCHITECT i2000SR and VITROS 5600 was $56.3 \mathrm{U} / \mathrm{mL}$. The agreement was excellent for CA 15-3 concentrations of $100 \mathrm{U} / \mathrm{mL}$ or less, whereas for higher CA 15-3 concentrations, the agreement was poorer.

\section{DISCUSSION}

CA 15-3 is the most commonly used tumor marker in breast cancer, although it is not specific to this

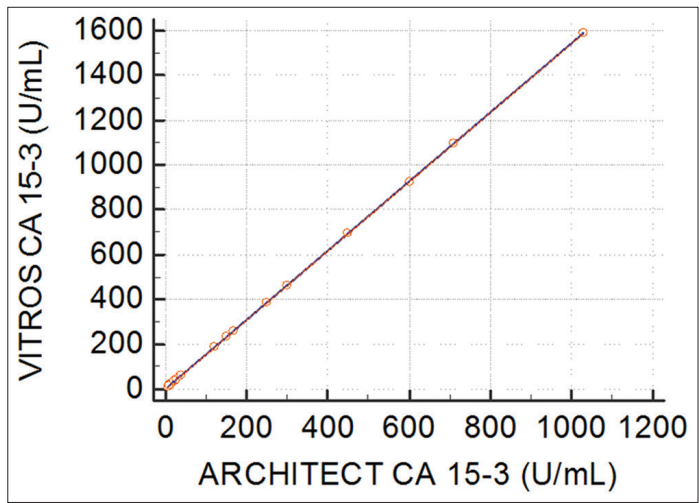

FIGURE 2. Comparison of cancer antigen (CA) 15-3 antigen determined in human serum samples using ARCHITECT i2000SR immunoassay analyzer and VITROS 5600 Integrated System $(r=1.00)$.

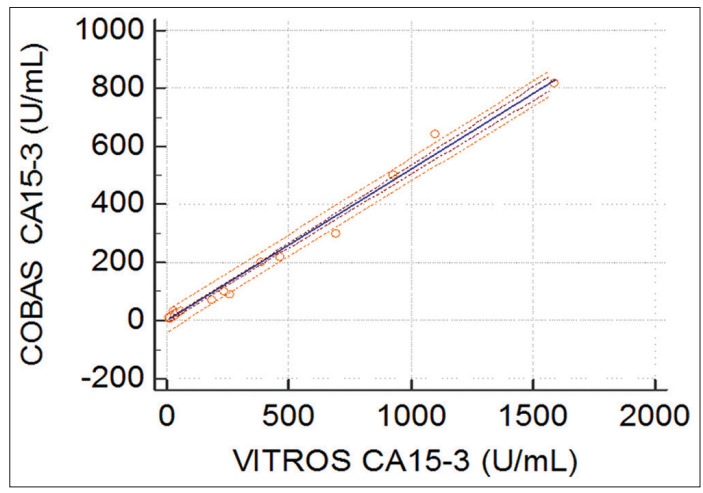

FIGURE 3. Comparison of cancer antigen (CA) 15-3 antigen determined in human serum samples using VITROS 5600 Integrated System and Cobas E 601 analyzer ( $r=0.9900$ to 0.9971$)$. cancer. The main non-malignant causes of elevated serum CA 15-3 levels are different hepatic or renal diseases. Results of different studies have shown moderately elevated serum CA 15-3 levels in a small percentage of patients with various disorders such as: Ovarian cysts, respiratory tract infections, and some autoimmune diseases $(14,15)$. Immunoassay testing has progressed over the years. Since the introduction of the first radioimmunoassay, several alternative and less hazardous detecting labels have been developed and the methods of antibody production have been improved. The analytical measurement ranges for the ARCHITECT i2000SR SR, VITROS 5600,

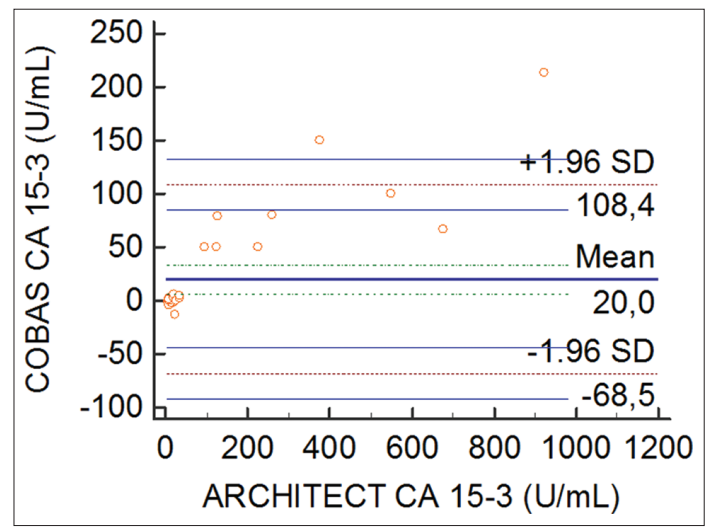

FIGURE 4. Comparison of two methods, ARCHITECT i2000SR and cobas e 601, for detection of cancer antigen (CA) 15-3. The bias (mean difference) is $20 \mathrm{U} / \mathrm{mL}$; $95 \%$ confidence interval [Cl], -68.5 to $108.4 \mathrm{U} / \mathrm{mL}$.

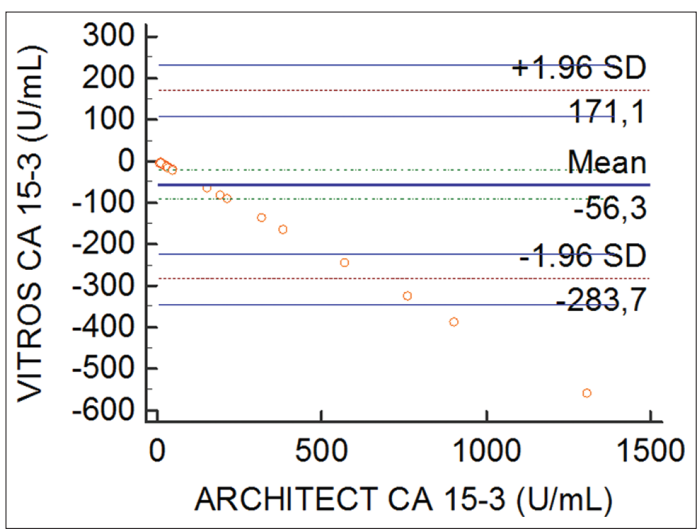

FIGURE 5. Comparison of two methods, ARCHITECT i2000SR and VITROS 5600, for cancer antigen (CA) 15-3 detection. The bias (mean difference) is $-56.3 \mathrm{U} / \mathrm{mL} ; 95 \%$ confidence interval [Cl], -283.7 to $171.1 \mathrm{U} / \mathrm{mL}$. 
and Cobas E 601 devices were 0-700 U/mL, $0-500 \mathrm{U} / \mathrm{mL}$, and $0-300 \mathrm{U} / \mathrm{mL}$, respectively. The ARCHITECT i2000SR method was selected as the comparison method because it has the largest analytical measurement range when compared with the other methods. Analyzing the Levey-Jennings report for CA 15-3 measurement by ARCHITECT, Cobas, and VITROS immunoassay technologies, we observed that the results were below two SD of the mean. The results of our study showed that the most precise method for determining serum CA 15-3 levels was the ARCHITECT i2000SR, with total coefficient of variation $(\mathrm{CV})$ less than $2.8 \%$, for both concentrations of the commercial control samples, and the reproducibility value less than $4.2 \%$. The Cobas E 601 technology showed the highest imprecision, with a total CV of $3.50 \%$ for the lowest concentration of the quality control samples. The reproducibility of the test results for all assays showed good CVs, ranging from 3.10 to $6.47 \%$. The recovery testing also showed good results for the ARCHITECT and Cobas methods, as well as for the VITROS method. The percent recoveries were $95-98 \%$ for Elecsys ${ }^{\circledR}$ CA 15-3 assay, 93-105\% for Vitros CA 15-3 assay, and 92-95\% for ARCHITECT CA 15-3 assay. The method comparison results showed a high correlation between the ARCHITECT and Cobas methods, with $\mathrm{r}$ values ranging from 0.9899 to 0.9971 and $p=0.0059$, as shown in Figure 1. The correlation coefficient for the VITROS and ARCHITECT methods was $r=1$ $(p=0.0027)$, as shown in Figure 2 . The study results also showed a high correlation between the VITROS and Cobas methods, with $\mathrm{r}$ ranging from 0.9900 to $0.9971(p=0.0029)$, as shown in Figure 3. The ARCHITECT i2000SR and VITROS 5600 methods have similar measurement ranges, 0-700 U/mL and $0-500 \mathrm{U} / \mathrm{mL}$ respectively; therefore, those methods showed a good coefficient of correlation and lower mean concentration differences. The method comparison results showed that the ARCHITECT i2000SR and VITROS 5600 had the highest correlation coefficient $(\mathrm{r}=1)$. Previous studies showed a good correlation between Abbott AxSym and Ortho-Clinical Vitros Eci systems in serum CA 15-3 level determination, with $\mathrm{r}=0.971$ (16). Our results showed a higher correlation coefficient between the ARCHITECT i2000SR and Cobas E 601 methods $(p=0.0059)$. The VITROS 5600 and Cobas E 601 showed a good correlation coefficient $(p=0.0029)$.

Among the causes of different results obtained with different assays are variations in instrument technology, assay design, and sample population. Different research groups presented comparisons and recovery results $(7,17)$. The ARCHITECT technology showed the largest analytical measurement range $(8.2-1030.2 \mathrm{U} / \mathrm{mL})$, followed by the VITROS (10.79-1010.96 U/mL), and the cobas e601 technology (5.9-817.4 U/mL). Similar analytical measurement ranges were reported in other studies (7).

Our results showed that four patients had CA 15-3 serum values above $35 \mathrm{Um} / \mathrm{L}$, measured by the VITROS 5600 Integrated System, and reference range values measured with the ARCHITECT i2000SR and Cobas E 601 technologies. One patient had CA $15-3$ serum value above $25 \mathrm{U} / \mathrm{mL}$ measured with the Cobas E 601 technology and reference range value measured with the VITROS 5600 and ARCHITECT i2000SR technologies. Despite high correlations observed, marked differences were also evident between the values obtained on different analyzers. Therefore, the usual recommendations on using the same immunoassay method, reagents, and analyzer during the follow-up of individual patients, and on defining new baseline concentrations when using different methods, should be followed for CA 15-3.

Our results could be explained by the fact that the ARCHITECT i2000SR has a higher analitical sensitivity (lower than $0.5 \mathrm{U} / \mathrm{mL}$ ), as compared to the Cobas E 601 (lower than $1 \mathrm{U} / \mathrm{mL}$ ). The limit of detection for CA 15-3 with the VITROS 5600 is also over $0.50 \mathrm{U} / \mathrm{mL}$. The cut-off value of tumor marker CA 15-3 differs between the different technologies, and this might cause variations in measured CA 15-3 levels. Our results showed that the highest average serum CA 15-3 concentration was determined by the Vitros CA 15-3 assay, as compared to the ARCHITECT CA 15-3 and Elecsys ${ }^{\circ}$ CA 15-3 assays, and that the mean differences between the methods ranged between $-20 \mathrm{U} / \mathrm{mL}$ to $56.3 \mathrm{U} / \mathrm{mL}$ (Figures 4 and 5). In general, lower values were obtained with the Elecsys ${ }^{\oplus}$ CA 15-3 assay, while the higher values were generally obtained 
with the Vitros CA 15-3 assay compared to the ARCHITECT method. Similar comparison method results were reported in other studies (7).

\section{CONCLUSION}

Tumor marker CA 15-3 in individual patients should be monitored using the same immunoassay and reagents on the same analyzer. Different immunoassay technologies quantify analytes of clinical interest using different monoclonal antibodies; the usage of antibodies with different specificities could explain differences in CA 15-3 serum values. Different antibodies recognize different parts of the molecule, and antigen heterogeneity may be partially responsible for inter-method differences. The substantial differences between the methods also indicate that defining new baseline and cut-off concentrations is necessary when using different methods and analyzers for measuring CA 15-3, during the follow-up of patients.

\section{CONFLICT OF INTEREST}

The authors declare no conflict of interest.

\section{REFERENCES}

1. Tobias R, Rothwell C, Wagner J, Green A, Liu YS. Development and evaluation of a radioimmunoassay for the detection of a monoclonal antibody defined breast tumor associated antigen 115D8/DF3. J Am Assoc Clin Chem 1985;31:986-92.

2. Hilkens J, Buijs F, Higers J, Hageman P, Calafat J, Sonnenberg A, et al. Monoclonal antibodies against human milk-fat globule membranes detecting differentiation antigens of the mammary gland and its tumors. Int $\mathrm{J}$ Cancer 1984;34(2):197-206.

https://doi.org/10.1002/ijc.2910340210.

3. Molina R, Auge JM, Farrus B, Zanon G, Pahisa J, Munoz M, et al. Prospective evaluation of carcinoembryonic antigen (CEA) and carbohydrate antigen 15.3 (CA 15.3) in patients with primary locoregional breast cancer. Clin Chem 2010;56(7):1148-57.

https://doi.org/10.1373/clinchem.2009.135566.
4. Molina R, Barak V, van Dalen A, Duffy MJ, Einarsson R, Gion H, et al Tumor markers in breast cancer - European Group on Tumor Markers recommendations. Tumour Biol 2005;26(6): 281-93.

https://doi.org/10.1159/000089260.

5. Sturgeon CM, Seth J. Why do immunoassays for tumour markers give differing results? - A view from the UK National External Quality Assessment Schemes. Eur J Clin Chem Clin Biochem 1996;34(9):755-9.

6. Sturgeon $\mathrm{C}$. Practice guidelines for tumor marker use in the clinic. Clin Chem 2002;48(8):1151-9.

7. Slev PR, Rawlins ML, Roberts WL. Performance characteristics of seven automated CA 15-3 assays. Am J Clin Pathol 2006;125(5):752-7.

https://doi.org/10.1309/G6X6PR7526FAKV0E.

8. Stern P, Bartos V, Vavrova J, Bezdickova D, Pechova M, Uhrova J, et al. Comparability of eight immunoassay procedures for the determination of CA 15-3 related markers. Clin Chem Lab Med 2003;41(8):1087-94.

https://doi.org/10.1515/CCLM.2003.168

9. Operations Manual CA 15-3 ARCHITECT SYSTEM Abbott Diagnostic REF 2K44-20 603-031 8/14/R07 B2K440, Abbott; 2012; 55.

10. Nafija Serdarević, Samira Mehanović. The possible role of tumor antigen CA 15-3, CEA and ferritin in malignant and benign disease. Journal of Health Sciences. 2012;2(2):138-143.

http://dx.doi.org/10.17532/jhsci.2012.52.

11. Molina R, Filella X, Auge M, Escudero JM. Clinical value of tumor markers - Current status and future prospects III. Roche Diagnostic International Ltd. 2013. p. 47-8.

12. K983690 - Vitros Immunodiagnostic Products Ca 15-3 Calibrators [published 1999 February 02]. Available from: https://pathsurveyor.com/510k/ K983690.

13. Tietz NW. Tietz textbook of clinical chemistry. $3^{\text {rd }}$ edition. Philadelphia, PA: B. Saunders Company; 1999.

14. Szekanecz E, Szucs G, Szekanecz Z, Tarr T, Antal-Szalmás P, Szamosi S et al. Tumor-associated antigens in systemic sclerosis and systemic lupus erythematosus: Associations with organ manifestations, immune laboratory markers and disease activity indices. J Autoimmun 2008;31(4):372-6. https://doi.org/10.1016/j.jaut.2008.08.008.

15. Ozsahin SL, Turgut B, Nur N, Dogan OT, Erselcan T, Berk S. Validity of the CA125 level in the differential diagnosis of pulmonary tuberculosis. Jpn $J$ Infect Dis 2008;61(1):68-9.

16. Klee G, Schreiber WE. MUC1 gene-derived glycoprotein assays for monitoring breast cancer (CA 15-3, CA 27.29, BR): Are they measuring the same antigen. Arch Pathol Lab Med 2004;128(10):1131-5.

17. Stern P, Bartos V, Vavrova J, Bezdickova D, Pechova M, Uhrova J, et al. Comparability of eight immunoassay procedures for the determination of CA 15-3 and related markers. Clin Chem Lab Med 2003;41(8):1087-94. https://doi.org/10.1515/CCLM.2003.168. 